\title{
Preoperative evaluation of dynamic contrast-enhanced MRI-guided lesion identification using morphometric and textural analysis for patients with epilepsy
}

\author{
DU FEIZHOU, GUAN JING, WANG PENG, WU JIALING and JIANG RUI \\ Department of Radiology, The General Hospital of Western Theater Command, Chengdu, Sichuan 610083, P.R. China
}

Received June 19, 2019; Accepted March 6, 2020

DOI: $10.3892 /$ etm.2020.9228

\begin{abstract}
Diagnosing epilepsy at the early stages is pivotal in the prevention and subsequent treatment of major epileptic events. MRI has been previously demonstrated to be beneficial in optimizing diagnostic efficacy and the subsequent treatment of epilepsy. In the present study, morphometric and textural analysis was performed pre-operatively on dynamic contrast-enhanced (Dce)-magnetic resonance imaging (MRI)-guided lesions in patients with epilepsy. The diagnostic efficacies of MRI and Dce-MRI were evaluated in 280 patients with epilepsy. The performance of Dce-MRI $(n=140)$ was compared with that of classic MRI $(n=140)$ in the analysis of the morphometric and textural features of the lesions, and the accuracy of mapping to regions of the brain that were potentially associated with the region of seizure onset was also investigated. Diagnostic quality was evaluated by comparing the signal-to-noise ratio (SNR) and contrast-to-noise ratio (CNR) of the hippocampus, the grey-white matter contrast and the morphometric and textural analysis of epileptogenic lesions. Data from the present study demonstrated that the regions of suspected epileptic activity in the brain were mapped more successfully using Dce-MRI compared with MRI. Image quality obtained by Dce-MRI was adequate for the detection of epileptic lesions, including those of focal cortical dysplasia or periventricular nodular heterotopia $(\mathrm{PNH})$. The diagnostic value of Dce-MRI for subtle lesions such as PNH was found to be more efficient compared with that of MRI due to the significantly increased SNR and CNR. In addition, Dce-MRI exhibited higher accuracy compared with MRI for the identification of cortical lesions and for the mapping to the zone of suspected seizure onset. Dce-MRI also exhibited higher sensitivity and specificity in the diagnosis of patients with epilepsy and mapping to the lesions associated with epilepsy
\end{abstract}

Correspondence to: Professor Jiang Rui, Department of Radiology, The General Hospital of Western Theater Command, 270 Rongdu Avenue, Chengdu, Sichuan 610083, P.R. China

E-mail: rui_jiangprof@outlook.com

Key words: epilepsy, diagnosis, MRI, dynamic contrast-enhanced MRI compared with MRI. These findings indicate that Dce-MRI is a feasible technique that may be beneficial in the diagnosis and subsequent management of patients with epilepsy.

\section{Introduction}

Epilepsy is a prevalent and devastating neurological disorder, which is characterized by recurrent, spontaneous and unprovoked seizures (1). The development and progression of epilepsy can be caused by extensive changes in gene transcription, resulting in aberrant remodeling of the neural network and hyperexcitability (2). Epilepsy can also originate from a wide range of disorders of the brain and nervous system, including tumors and non-neoplastic lesions (3-5). Currently, a number of studies have demonstrated that epigenetic mechanisms, including post-translational modifications, non-coding RNA and chemical modifications to the DNA, exert significant influence on the pathogenesis of epilepsy (6-8). Following these observations, combined with reports implicating the possible involvement of epigenetic mechanisms in the regulation of metabolism underlying the neurological activities of epilepsy, the potential for the use of epigenetic markers in epilepsy diagnosis and prognosis has been evaluated (9-11). Collectively, based on the characteristics of epilepsy, the aforementioned findings may serve as a guide for the diagnosis of epilepsy and the development of novel therapeutic strategies for epilepsy prevention or intervention.

Clinically, multiple types of diagnoses and the diverse set of clinical presentations that are associated with epilepsy render accurate diagnosis and treatment difficult (12). Epilepsy is frequently defined as a disease that is characterized by one or more seizures with genetic etiologies (13). Electroencephalography is currently the most commonly applied method for assessing epilepsy, which facilitates diagnosis, the classification of seizure types, localization of the pathological area, designation of treatment strategies and monitoring of prognosis (14). Magnetic resonance imaging (MRI) is a particularly useful technique for the evaluation of seizure etiology and the identification of potential seizure onset sites (15-17). Previous MRI data suggests that epilepsy is caused by parenchymal atrophy that is disproportionate for age, and is often associated with aberrant hyperactivity in the cortical/subcortical T2 region of the brain (18). MRI serves a crucial role in the routine diagnosis of epilepsy and contributes 
to etiological diagnosis in general clinical neuroscience. Additionally, MRI data is widely applied in post-processing procedures for the analysis of co-registered, three-dimensional volumes from images obtained using various modalities (19). Dynamic contrast-enhanced MRI (Dce-MRI) exhibits significant diagnostic quality in determining the preoperative localization of epileptic foci and evaluating prognosis in patients with epilepsy (20). Textural analysis of MRI images also facilitates quantification during the imaging assessment of epilepsy, which has been previously found to be essential in understanding juvenile myoclonic epilepsy (21). Morphometric and textural analysis of MRI images allows the detection of lesions in pediatric epilepsy (22). Therefore, it is of importance to review the preoperative evaluation processes of Dce-MRI-guided lesions using morphometric and textural analysis for patients with epilepsy.

In the present study, a quantitative evaluation was performed on the signal-to-noise ratio (SNR) and contrast-to-noise ratio (CNR) yielded using Dce-MRI or MRI from hippocampal images obtained from patients with epilepsy. Grey-white matter contrast (GWMC) and epileptogenic lesion (morphometric and textural analysis) images taken using MRI and Dce-MRI from patients with epilepsy were also analyzed. Additionally, images of epileptogenic lesions acquired using MRI and Dce-MRI and the corresponding diagnostic efficacy in patients with epilepsy were compared.

\section{Materials and methods}

Participants and clinical scoring. A total of 280 patients with epilepsy (146 females and 134 males; mean age, $34.7 \pm 13.5$ years) and 166 healthy controls (male/female, $86 / 80$; mean age, $33.8 \pm 8.4$ years) were recruited at The General Hospital of Western Theater Command (Chengdu, China) between June 2016 and July 2018. The healthy controls were individuals who received diagnosis using MRI $(n=83)$ and Dce-MRI $(n=83)$. Information on the presence of active or calcified lesions was recorded in each patient by radiological examination based on MRI examinations. Epilepsy in patients was diagnosed using MRI $(n=140)$ or Dce-MRI $(n=140)$. The type of imaging used was according to the patient's preference. The exclusion criteria for all participants were as follows: i) Patients with a history of follow-up due to neurotuberculosis, neurotoxoplasmosis, tuberous sclerosis and surgery for temporal lobe epilepsy; and ii) patients with brain tumor, Parkinson's disease or physical brain damage. A schematic workflow of the present study is shown in Fig. 1. Engel classification (23) was applied for grading epilepsy.

MRI and Dce-MRI. Patients with epilepsy were imaged using a Philips Intera Achieva 3.0T MRI scanner (Philips Medical Systems B.V.). Acquisitions were obtained in the coronal, sagittal and axial planes, with coronal sections also obtained perpendicularly along the axis of the hippocampal formation. MRI was conducted using the following parameters: i) Repetition time, $5 \mathrm{msec}$; ii) echo time, $2 \mathrm{msec}$; iii) matrix, $212 \times 213$; iv) flip angle, $90^{\circ}$; v) field of view,

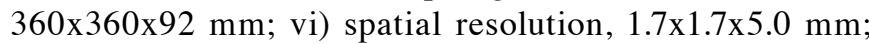
vii) number of slices, 19; viii) average number of signals, 1 ; ix) temporal resolution, $8.3 \mathrm{sec}$; $\mathrm{x}$ ) acquisition time, $8.5 \mathrm{~min}$; and $\mathrm{xi}$ ) number of dynamic scans, 60. For Dce-MRI, a bolus of $0.10 \mathrm{mmol} / \mathrm{kg}$ gadobutrol (Gadovist; Bayer AG) was intravenously injected into each patient followed by a $20-\mathrm{ml}$ saline flush. The subsequent MRI examination consisted of turbo spin-echo T1- and T2-weighted sequences and a three-dimensional DCE sequence. Details of sequence parameters were as described previously (24). For Dce-MRI examinations, MR images were obtained using the following parameters: i) Repetition time, 1,200 msec; ii) echo time, 27 msec; iii) matrix, 212x213; iv) flip angle, $90^{\circ}$; v) field of view, 360x360x92 mm; vi) spatial resolution, 1.7x1.7x5.0 mm; vii) number of slices, 19; viii) average number of signals, 1 ; ix) temporal resolution, $8.3 \mathrm{sec}$; $\mathrm{x}$ ) acquisition time, $8.5 \mathrm{~min}$; xi) number of dynamic scans, 60; xii) continuous phase, 24; xiii) time resolution, $2 \mathrm{sec}$; and xiv) scan time, $48 \mathrm{sec}$. The MRI images of epileptic lesions were retrospectively evaluated by two board-certified radiologists. In cases of disagreements between the two radiologists regarding the diagnosis of the same patient, pathological data were referred to a different pair of radiologists for the final diagnostic decision. Electrocorticography and pathological results were considered as the gold standard for the identification and localization of pathogenic foci and diagnostic consistency (25), in addition to the comparison of diagnostic efficacy between Dce-MRI and MRI. Patients with diseases including epilepsy, Tourette's syndrome, hypoglycemia and syncope were excluded from the healthy control. All patients with epilepsy were definitively confirmed by three independent epilepsy specialists. The locations of epileptic lesions in MRI and Dce-MRI images were calculated and analyzed using FrameLink software version 1.0 (Medtronic).

MRI image analysis. All MRI images were transferred to a computer-aided diagnostic system (Merge CADstream ${ }^{\circledR}$; version 4.1; IBM Corp.) and analyzed by two pathologists who specialized in epilepsy. Signal intensity in each pixel was recorded in all patients. Colors were assigned according to changes in pixel values following contrast injection. The computer-aided diagnostic system report was prospectively saved by a research assistant, where parameters including peak signal intensity (\%), SNR and CNR of the hippocampus, area of the GWMC and epileptogenic lesions were recorded in the MRI image analysis. MRI and Dce-MRI data were analyzed using IDL ${ }^{\circledR}$ software version 2.2 (Exelis VIS; Harris Geospatial Solutions, Inc.) by applying a modified Brix's linear two-compartment pharmacokinetic model to determine the SNR, CNR and GWMC (26).

Statistical analysis. Measurement data are expressed as the mean \pm standard deviation, whilst count data are presented as n (\%). Statistical analysis of clinical data was performed using SPSS 22.0 software (IBM Corp.). Normally distributed data were analyzed using independent samples t-test whereas non-normally distributed data were analyzed using the Mann-Whitney $U$ test. Univariate analysis for enumeration data was performed using $\chi^{2}$ test. A receiver operating characteristic (ROC) curve was generated to evaluate the diagnostic value of MRI and Dce-MRI for differentiating patients with epilepsy from healthy controls. $\mathrm{P}<0.05$ was considered to indicate a statistically significant difference. 


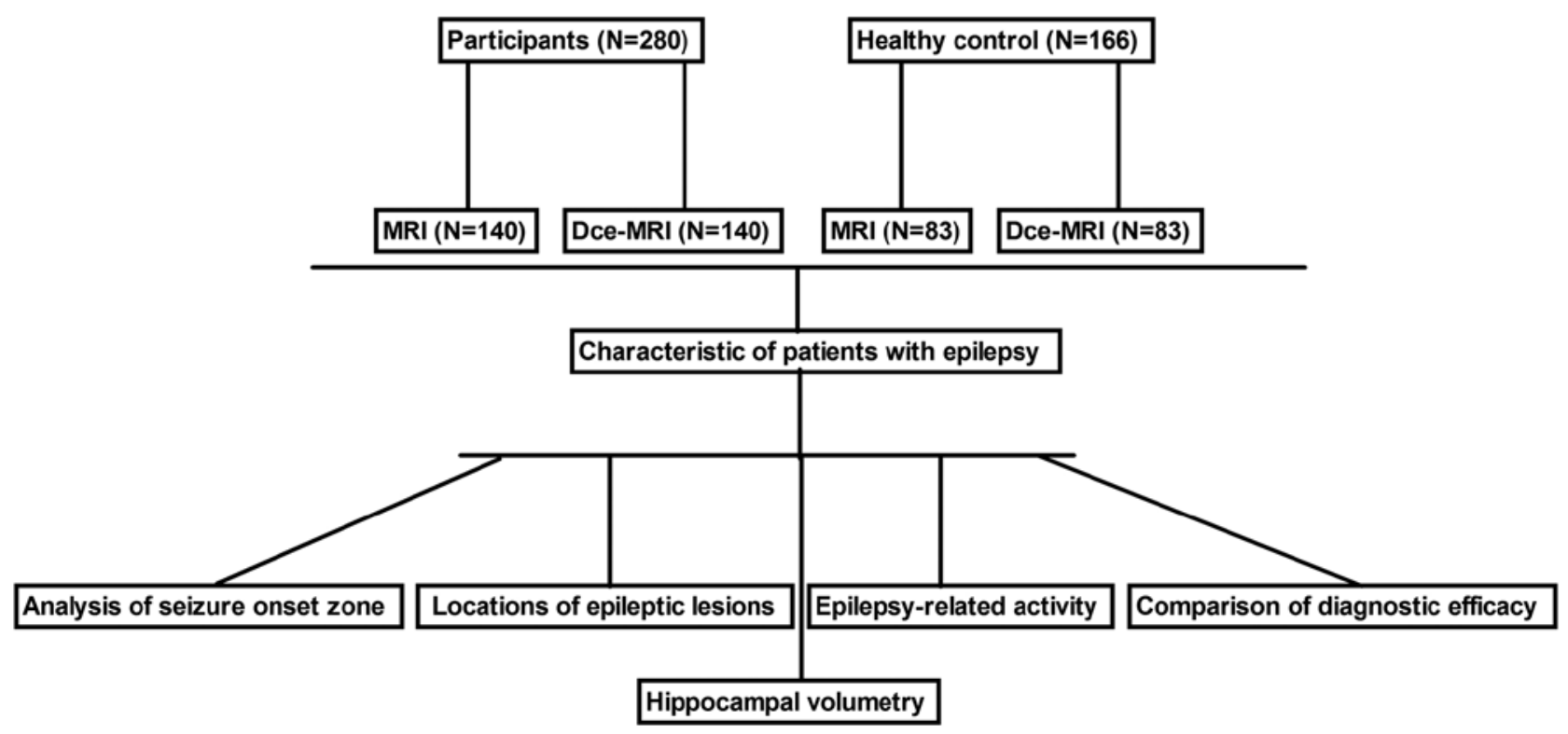

Figure 1. Schematic of the workflow in the present study. MRI, magnetic resonance imaging; Dce-MRI, dynamic contrast-enhanced MRI.

Table I. Clinical, demographic and neuropsychological data of patients with epilepsy.

\begin{tabular}{lccc}
\hline Patient characteristic & MRI & Dce-MRI & P-value \\
\hline Age (years) & $34.7 \pm 13.5$ & $36.4 \pm 11.2$ & 0.76 \\
Sex (female/male) & $76 / 64$ & $70 / 70$ & 0.80 \\
Suspected zone of seizure onset & & & \\
Temporal & 40 & 45 & 0.62 \\
Parietal & 42 & 40 & 0.82 \\
Frontal/Temporal & 25 & 21 & 0.72 \\
Anterior Temporal & 14 & 17 & 0.58 \\
Frontal & 15 & 12 & 0.63 \\
Unknown & 4 & 5 & 0.88 \\
Antiepileptic drugs & & & 0.75 \\
Phenytoin & 24 & 26 & 0.58 \\
Pregabalin & 28 & 32 & 0.66 \\
Primidone & 25 & 23 & 0.56 \\
Retigabine & 24 & 28 & 0.64 \\
Rufinamide & 32 & 30 & \\
\hline
\end{tabular}

Data are presented either as number or mean \pm standard deviation. Statistical analysis was performed using independent samples $\mathrm{t}$-test, Mann-Whitney U test or the $\chi^{2}$ test.

\section{Results}

Characteristics of patients with epilepsy. Table I provides the clinical and demographic details of the patients with epilepsy. A total of 280 patients with epilepsy were included, who were imaged using Dce-MRI or MRI for morphometric and textural analysis. The ability of classic MRI $(n=140)$ and Dce-MRI $(n=140)$ to map to the regions of suspected seizure onset in the brain were compared. Among the 280 patients included in the present study, 50 were treated with phenytoin, 60 with pregabalin, 48 with primidone, 52 with retigabine and 62 with rufinamide. No significant differences were observed in the baseline characteristics of patients with epilepsy between the MRI and Dce-MRI groups.

Evaluation of locations of epileptic lesions. Dce-MRI revealed markedly clearer morphometric and textural features of the lesions compared with MRI in patients with epilepsy (Fig. 2). The morphometric analysis showed that the epileptic lesions were predominantly localized in the parietal, temporal, frontal/temporal, anterior temporal or frontal lobes in patients with epilepsy, where the shape of the parietal lobes 
A
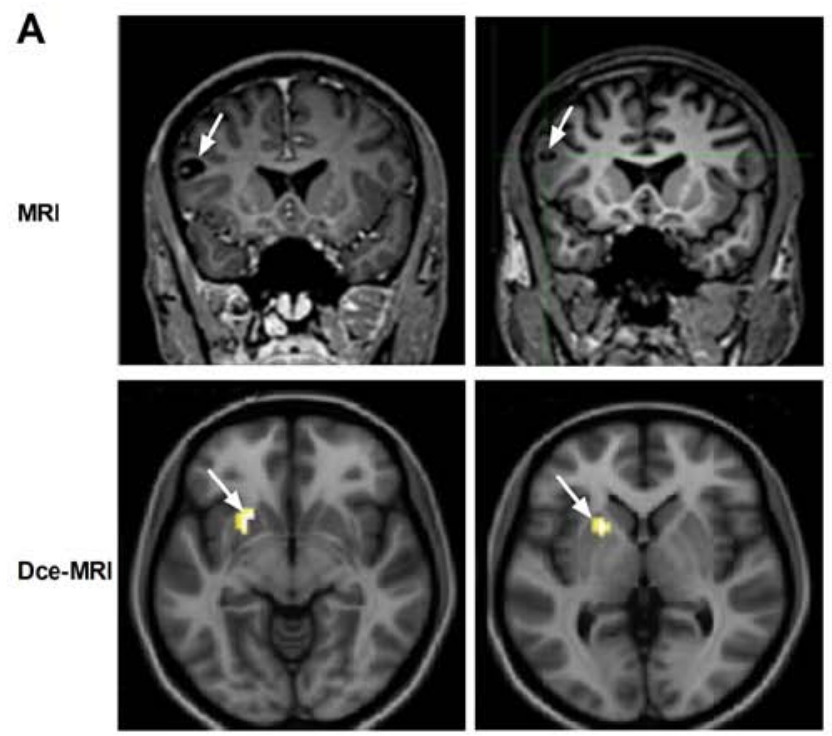

B
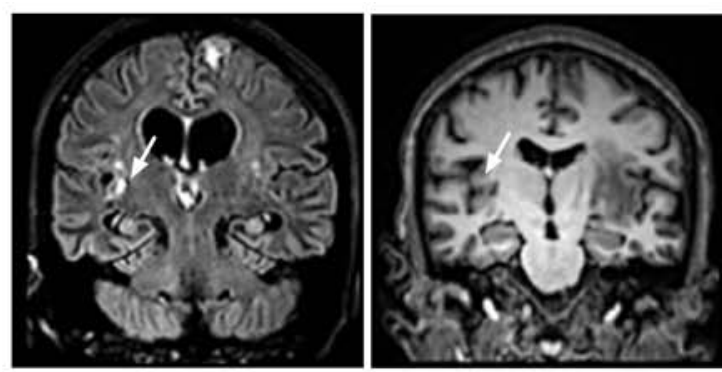

MRI

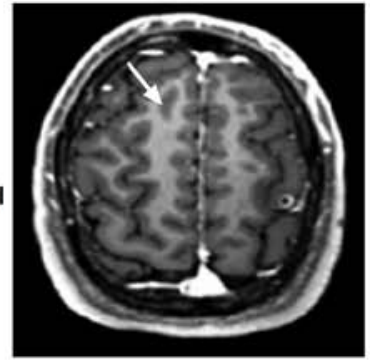

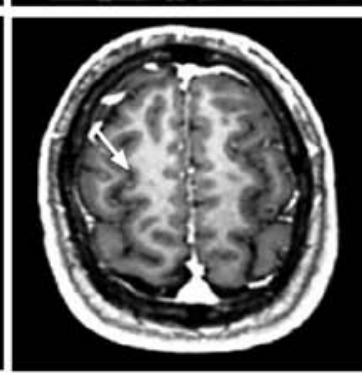

Figure 2. Evaluation of the localization of epileptic lesions using MRI or Dce-MRI from the same patients. (A) Morphometric analysis of lesions in patients with epilepsy diagnosed using MRI or Dce-MRI. (B) Textural analysis of lesions in patients with epilepsy diagnosed using MRI or Dce-MRI. MRI, magnetic resonance imaging; Dce-MRI, dynamic contrast-enhanced MRI.

Table II. Magnitude of epileptic injuries in epileptic patients in the MRI and Dce-MRI groups.

\begin{tabular}{lll}
\hline Magnitude of epileptic injury & MRI & Dce-MRI \\
\hline Grade I & $51(36.7)$ & $55(39.2)$ \\
Grade II & $55(39.6)$ & $51(36.3)$ \\
Grade III & $18(12.5)$ & $22(15.8)$ \\
Grade IV & $16(11.2)$ & $12(8.7)$ \\
\hline
\end{tabular}

Data are presented as number $(\%)$. Dce-MRI, dynamic contrastenhanced MRI.

was atrophic. Textural analysis revealed increasing variations in spatial arrangements in the hippocampal region, indicative of progressive microstructural heterogeneity. The magnitudes of epileptic injuries in patients with epilepsy in the MRI and Dce-MRI groups are shown in Table II. The grading system for epilepsy was determined using the criteria from the American Academy of Neurology Practice Parameter (27). These data suggest that the ability of Dce-MRI to identify lesions in patients with epilepsy is more efficient compared with that of MRI.

Diagnostic efficacy of Dce-MRI for focal cortical dysplasia in patients with epilepsy. To verify the diagnostic efficacy of Dce-MRI, ROC analysis was performed to compare the diagnostic efficacy of Dce-MRI and MRI for focal cortical dysplasia in patients with epilepsy (Fig. 3A). Dce-MRI exhibited higher diagnostic accuracy [area under the curve (AUC), 0.910; 95\% CI, 0.868-0.946] compared with MRI (AUC, 0.852; 95\% CI, $0.810-0.906)$. Using the cutoff value of 1.764 for Dce-MRI, the optimal sensitivity and specificity were calculated to be 93.2 and 94.5\% respectively. In addition, Dce-MRI also demonstrated higher accuracy compared with MRI for the identification of cortical lesions and the successful mapping of images to the suspected zone of seizure onset (Fig. 3B). In addition, Dce-MRI increased diagnostic quality by significantly increasing the SNR and CNR, and revealed the areas of GWMC and epileptogenic lesions more clearly compared with MRI (Fig. 3C-F). These data suggest that Dce-MRI is a reliable method for the diagnosis of epileptic lesions in patients with epilepsy.

Analysis of patients with active lesions using Dce-MRI. Compared with patients imaged using MRI, those imaged using Dce-MRI demonstrated increased activity in the left inferior frontal gyrus (Fig. 4A). Dce-MRI revealed higher numbers of active epileptic lesions in the whole brain compared with MRI (Fig. 4B). The outcomes demonstrated that brain activities associated with epilepsy were completely and clearly displayed by Dce-MRI compared with MRI (Fig. 4C). Dce-MRI presented with higher degrees of accuracy in locating epileptic lesions compared with MRI, supported by observations that the image quality of Dce-MRI was adequate for the detection of the epileptogenic lesions of periventricular nodular heterotopia (PNH; Fig. 4D). These observations suggest that Dce-MRI can evaluate the location and characteristics of epileptic pathology in patients with epilepsy.

\section{Discussion}

The pathophysiology of epilepsy includes the simultaneous presence of hippocampal sclerosis and its comorbidities associated with psychiatric and cognitive complications (28). Clinically, MRI is routinely performed to diagnose and assess non-lesional cingulate epilepsy $(19,29,30)$. Dce-MRI examination has previously demonstrated considerable accuracy in the preoperative localization of epileptic foci, suggesting that Dce-MRI is suitable for use as a basic reference for the diagnosis of patients with epilepsy (20). In the present study, the diagnostic efficacy and potency of the Dce-MRI 

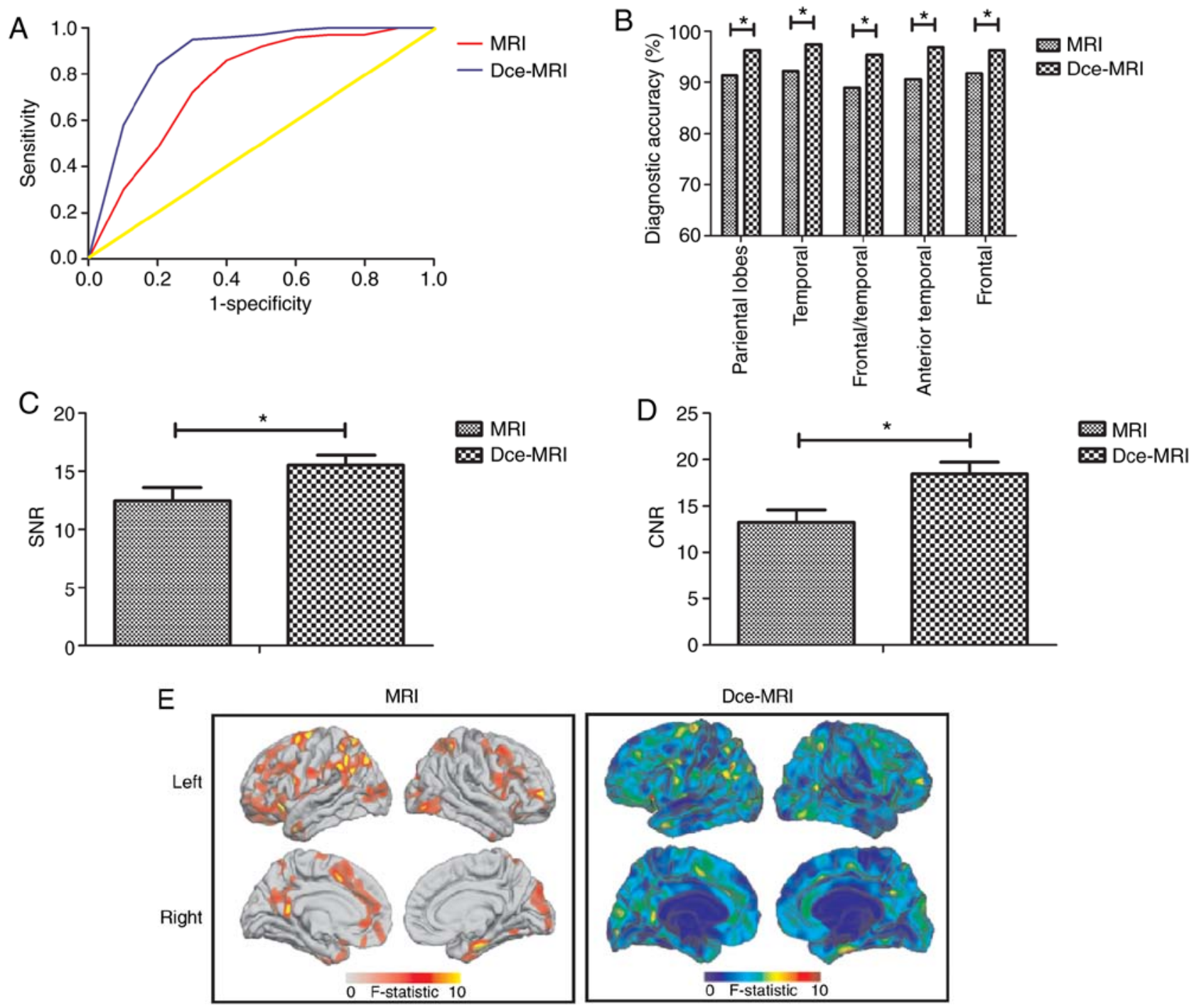

F
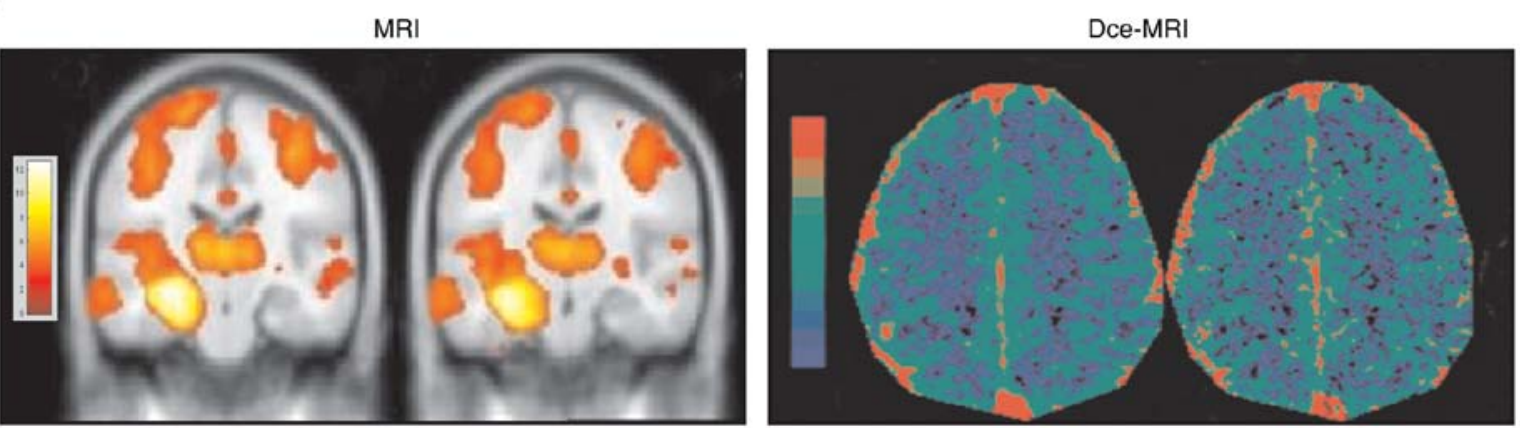

Figure 3. Diagnostic efficacy of Dce-MRI for focal cortical dysplasia in patients with epilepsy. (A) Comparison of diagnostic sensitivity and specificity of MRI and Dce-MRI for epilepsy. (B) Comparison of diagnostic accuracy of MRI and Dce-MRI in the identification of cortical lesions and the imaging findings co-localized to the zone of suspected seizure onset. Statistical analysis was performed using $\chi^{2}$ test. "P<0.05. (C) SNR and (D) CNR from images of the hippocampus were used to compare the diagnostic quality of MRI and Dce-MRI. ${ }^{*} \mathrm{P}<0.05$. Data was analyzed using the Mann-Whitney U test. (E) Images showing the area of GWMC and (F) epileptogenic lesions obtained from patients with epilepsy using MRI and Dce-MRI. SNR, signal-to-noise ratio; CNR, contrast-to-noise ratio; GWMC, grey-white matter contrast; MRI, magnetic resonance imaging; Dce-MRI, dynamic contrast-enhanced MRI.

platform was comprehensively investigated, and the results demonstrated that Dce-MRI can be applied to identify and locate pathological lesions in cases of epilepsy.

Focal cortical dysplasia is the major histopathological type among patients with epilepsy who require surgical resection, and previous data suggest that the MRI diagnostic platform provides essential information for the detection of focal cortical dysplasia (31). Morphometric analysis is based on the most common features of focal cortical dysplasia, including cortical thickness and the blurring of the grey-white matter interface (32). The application of structural MRI emphasizes the unique role of this non-invasive technique, which enables 
A

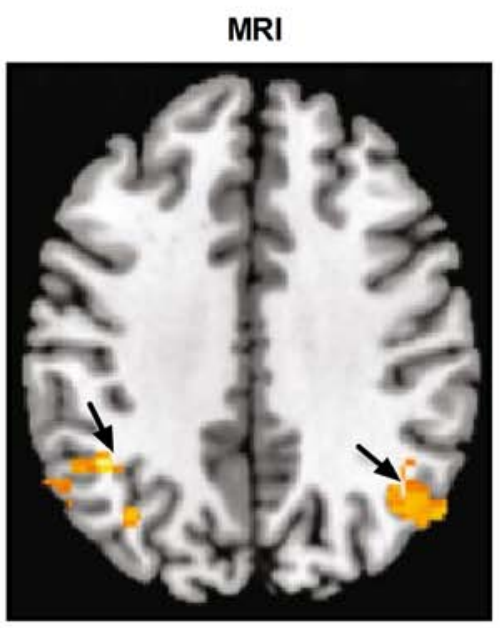

Dce-MRI

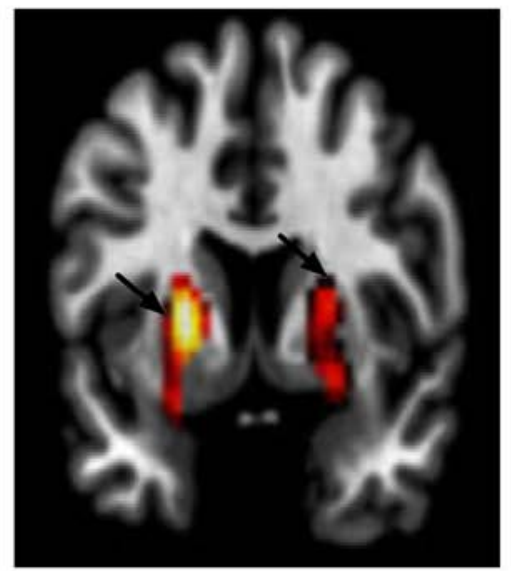

B

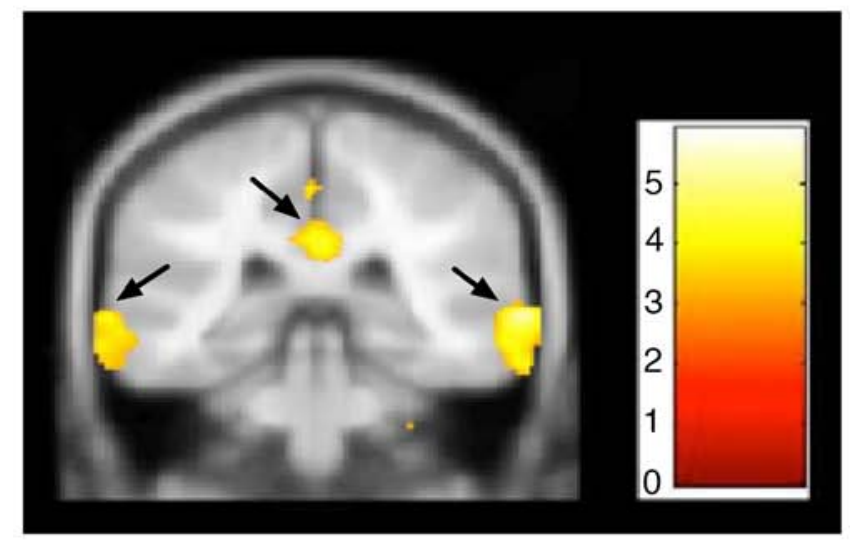

C

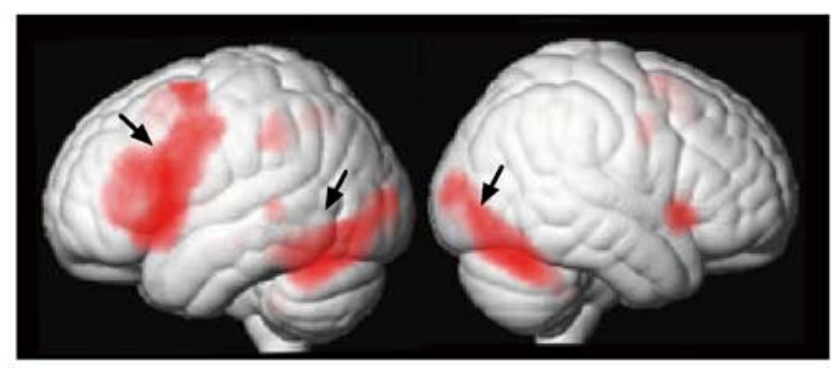

MRI

D

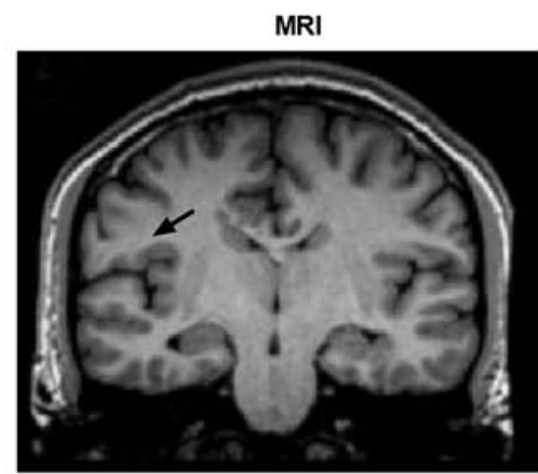

Dce-MRI

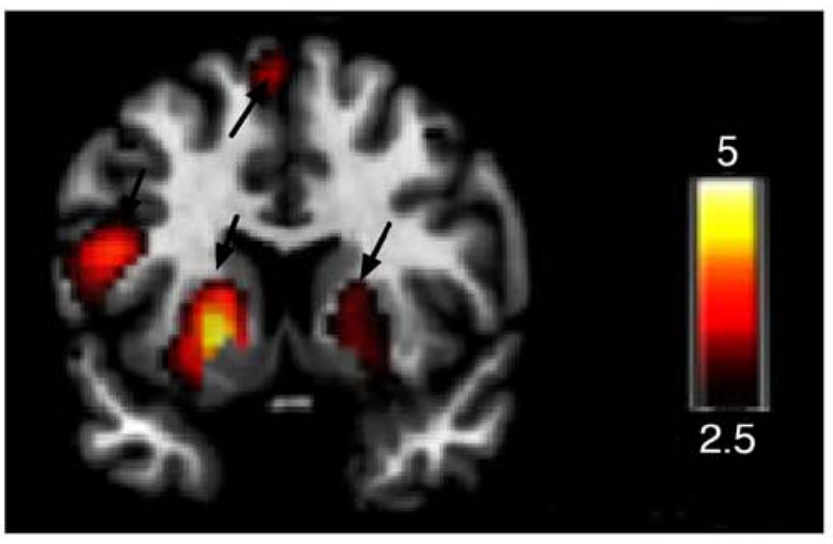

Dce-MRI

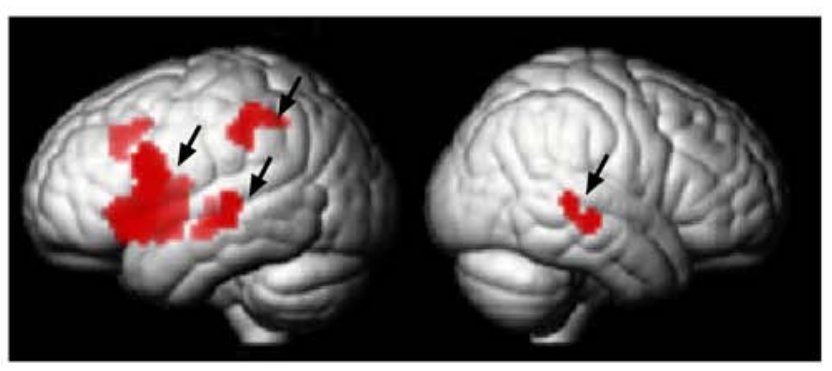

Dce-MRI

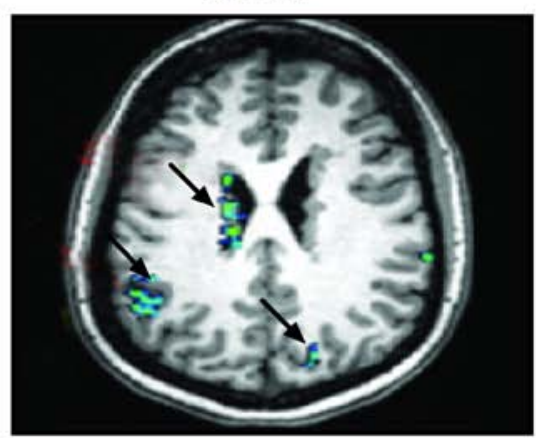

Figure 4. Analysis of active lesions using Dce-MRI in patients with epilepsy. (A) Comparison of activity in the left inferior frontal gyrus of patients with epilepsy between the MRI and Dce-MRI groups. (B) Comparison of active epileptic lesions in whole brain images of patients with epilepsy between the MRI and Dce-MRI groups. (C) Location of brain activities associated with epilepsy as mapped using MRI and Dce-MRI in patients with epilepsy. (D) Images of PNH epileptogenic lesions in patients with epilepsy in the MRI and Dce-MRI groups. PNH, periventricular nodular heterotopia; MRI, magnetic resonance imaging; Dce-MRI, dynamic contrast-enhanced MRI. 
the location of focal regions that are potentially associated with epilepsy and the designation of management strategies for patients with epilepsy (33). In the present study, the locations of epileptic lesions in patients were clearly identified and analyzed using Dce-MRI. Compared with MRI, images obtained by Dce-MRI exhibited higher resolution, which clearly displayed the minimal abnormal lesions in the brain, clarified the primary localization of epileptic foci and detected the abnormal structures in the brain (20). Additional data obtained, including the morphometric/textural lesions and signal intensity, showed that the epileptic lesions were primarily located in the parietal lobes of patients with epilepsy where the shape was abnormal. It is therefore of importance to recognize that epileptic features and outcomes as in the present study suggest the improved sensitivity of identifying an abnormality using Dce-MRI.

Measurements from MRI images can be applied for the identification of epileptic regions and size estimation for clinical reference (34-36). Resting functional MRI data analysis using temporal clustering is an efficient method, which can assist clinical practice in characterizing the epileptogenic network in patients with epilepsy (37). In the present study, Dce-MRI exhibited higher diagnostic sensitivity and sensitivity for epilepsy compared with MRI. In addition, Dce-MRI demonstrated higher accuracy in identifying cortical lesions compared with MRI, since the imaging findings co-localized to the diagnosed zone of suspected seizure onset, the SNR and CNR were increased in images of the hippocampus, and the areas of the GWMC and epileptogenic lesions were increased. Reorganization of the brain may occur in patients with epilepsy prior to treatment and/or surgery; therefore, leaving more regions of the brain and GWMC intact provides a foundation for functional recovery following diagnosis. Additionally, patients diagnosed with epilepsy using Dce-MRI presented with higher activity on the left inferior frontal gyrus, higher numbers of active lesions and PNH compared with those diagnosed using MRI. These findings suggest that epileptic features in the brain can be readily detected using Dce-MRI.

Limitations associated with the present study concern the study population, follow-up after diagnosis and the representativeness of the results. The sample size of the cases of epilepsy investigated was relatively small, meaning that further studies using larger sample sizes would be required to determine the diagnostic efficacy and recognized standardization of Dce-MRI procedures in patients with epilepsy. In addition, the present study did not include long-term follow-up data following epilepsy diagnosis. The present study design also did not include a comparison between MRI and Dce-MRI on a single patient, rendering impossible the establishment of intraclass correlation coefficients, which could be used to estimate the degree to which both imaging methods match the parameters in patients with epilepsy. In the future, multi-center studies should be conducted, which should include feasibility of outcomes analyses, in addition to analyzing further pathological details in a larger cohort to obtain data that can adequately represent the general population of patients with epilepsy.

In conclusion, diagnostic data obtained using Dce-MRI in the present study suggest that Dce-MRI is a promising diagnostic approach for minimizing cognitive morbidity associated with open surgical resection for treating epilepsy. Dce-MRI can detect epileptic lesions, aid in the identification of pathological features and locate focal cortical dysplasia. By identifying the potential regions of suspected epileptic activity in the brain using Dce-MRI, clinicians can design strategies of therapeutic intervention using data obtained from Dce-MRI.

\section{Acknowledgements}

Not applicable.

\section{Funding}

The present study was supported by Project of Sichuan Science and Technology Department (grant no. 2018JY0604).

\section{Availability of data and materials}

The datasets used and/or analyzed during the current study are available from the corresponding author on reasonable request.

\section{Authors' contributions}

DF, GJ and WP performed the experiments. WJ analyzed the data. JR designed the study and wrote the manuscript.All authors read and approved this article.

\section{Ethics approval and consent to participate}

This study was approved by the Ethics Committee of The General Hospital of Western Theater Command (approval no. 55942116.5.0000.5404). Signed and written informed consent was provided by all participants.

\section{Patient consent for publication}

Patients consented to the publication of their MRI images.

\section{Competing interests}

The authors declare that they have no competing interests.

\section{References}

1. Veersema TJ, Swampillai B, Ferrier CH, van Eijsden P, Gosselaar PH, van Rijen PC, Spliet WGM, Mühlebner A, Aronica E and Braun KPJ: Long-term seizure outcome after epilepsy surgery in patients with mild malformation of cortical development and focal cortical dysplasia. Epilepsia Open 4: 170-175, 2018.

2. Auvin S, Wirrell E, Donald KA, Berl M, Hartmann H, Valente KD, Van Bogaert P, Cross JH, Osawa M, Kanemura H, et al: Systematic review of the screening, diagnosis, and management of ADHD in children with epilepsy. Consensus paper of the task force on comorbidities of the ILAE pediatric commission. Epilepsia 59: 1867-1880, 2018

3. Vaughan KA, Lopez Ramos C, Buch VP, Mekary RA, Amundson JR, Shah M, Rattani A, Dewan MC and Park KB: An estimation of global volume of surgically treatable epilepsy based on a systematic review and meta-analysis of epilepsy. J Neurosurg 1-15: 2018.

4. Verche E, San Luis C and Hernandez S: Neuropsychology of frontal lobe epilepsy in children and adults: Systematic review and meta-analysis. Epilepsy Behav 88: 15-20, 2018. 
5. Kwok SC: Website review: Paediatric epilepsy network of new south Wales. J Paediatrics Child Health 54: 1045, 2018.

6. Zhao H, Lin G, Shi M, Gao J, Wang Y, Wang H, Sun H and Cao Y: The mechanism of neurogenic pulmonary edema in epilepsy. J Physiol Sci 64: 65-72, 2014.

7. Tian XB, Li RC, Bu HL, Liu C, Liu TT, Xiang HB and Lu CJ: The mechanism of electroacupuncture for predicting the efficacy of deep brain stimulation in pharmacoresistant epilepsy may be involved in the melanocortinergic signal. Epilepsy Behav 29: 594-596, 2013.

8. Cho $\mathrm{CH}$ : New mechanism for glutamate hypothesis in epilepsy. Front Cell Neurosci 7: 127, 2013.

9. Middlebrooks EH, Grewal SS, Stead M, Lundstrom BN Worrell GA and Van Gompel JJ: Differences in functional connectivity profiles as a predictor of response to anterior thalamic nucleus deep brain stimulation for epilepsy: A hypothesis for the mechanism of action and a potential biomarker for outcomes. Neurosurg Focus 45: E7, 2018.

10. Beckonert NM, Opitz T, Pitsch J, Soares da Silva P and Beck H: Polyamine modulation of anticonvulsant drug response: A potential mechanism contributing to pharmacoresistance in chronic epilepsy. J Neurosci 38: 5596-5605, 2018.

11. Viswanatha GL, Venkataranganna MV, Prasad NBL and Godavarthi A: Achyranthes aspera attenuates epilepsy in experimental animals: Possible involvement of GABAergic mechanism. Metab Brain Dis 32: 867-879, 2017.

12. St Louis EK and Cascino GD: Diagnosis of epilepsy and related episodic disorders. Continuum (Minneap Minn) 22: 15-37, 2016.

13. Helbig KL, Farwell Hagman KD, Shinde DN, Mroske C, Powis Z, Li S, Tang S and Helbig I: Diagnostic exome sequencing provides a molecular diagnosis for a significant proportion of patients with epilepsy. Genet Med 18: 898-905, 2016.

14. Carmichael DW, Thornton JS, Rodionov R, Thornton R, McEvoy A, Allen PJ and Lemieux L: Safety of localizing epilepsy monitoring intracranial electroencephalograph electrodes using MRI: Radiofrequency-induced heating. J Magn Reson Imaging 28: 1233-1244, 2008.

15. Brown MG, Drees C, Nagae LM, Thompson JA, Ojemann S and Abosch A: Curative and palliative MRI-guided laser ablation for drug-resistant epilepsy. J Neurol Neurosurg Psychiatry 89: 425-433, 2018

16. Drane DL: MRI-Guided stereotactic laser ablation for epilepsy surgery: Promising preliminary results for cognitive outcome. Epilepsy Res 142: 170-175, 2018.

17. Delev D, Quesada CM, Grote A, Boström JP, Elger C, Vatter H and Surges R: A multimodal concept for invasive diagnostics and surgery based on neuronavigated voxel-based morphometric MRI postprocessing data in previously nonlesional epilepsy. J Neurosurg 128: 1178-1186, 2018

18. Fredriksen JR, Carr CM, Koeller KK, Verdoorn JT, Gadoth A, Pittock SJ and Kotsenas AL: MRI findings in glutamic acid decarboxylase associated autoimmune epilepsy. Neuroradiology 60: 239-245, 2018

19. Ruber T, David B and Elger CE: MRI in epilepsy: Clinical standard and evolution. Curr Opin Neurol 31: 223-231, 2018.

20. Wang GB, Long W, Li XD, Xu GY and Lu JX: Dynamic contrast-enhanced magnetic resonance imaging (DCE-MRI) combined with positron emission tomography-computed tomography (PET-CT) and video-electroencephalography (VEEG) have excellent diagnostic value in preoperative localization of epileptic foci in children with epilepsy. Med Sci Monit 23: 1-10, 2017.

21. de Oliveira MS, Betting LE, Mory SB, Cendes F and Castellano G: Texture analysis of magnetic resonance images of patients with juvenile myoclonic epilepsy. Epilepsy Behav 27: 22-28, 2013

22. Kulaseharan S, Aminpour A, Ebrahimi M and Widjaja E: Identifying lesions in paediatric epilepsy using morphometric and textural analysis of magnetic resonance images. Neuroimage Clin 21: 101663, 2019.

23. Iachinski RE, de Meneses MS, Simão CA, da Rocha SF, de Oliveira Braga F and Kowacs PA: Patient satisfaction with temporal lobectomy/selective amygdalohippocampectomy for temporal lobe epilepsy and its relationship with Engel classification and the side of lobectomy. Epilepsy Behav 31: 377-380, 2014
24. Chen L, Ye Y, Chen H, Chen S, Jiang J, Dan G and Huang B Dynamic contrast-enhanced magnetic resonance imaging for differentiating between primary tumor, metastatic node and normal tissue in head and neck cancer. Curr Med Imaging Rev 14: 416-421, 2018.

25. Boling W: Diagnosis and surgical treatment of epilepsy. Brain Sci 8: E115, 2018.

26. Rösch J, Hamer HM, Mennecke A, Kasper K, Engelhorn T, Doerfler A and Graf W: 3T-MRI in patients with pharmacoresistant epilepsy and a vagus nerve stimulator: A pilot study. Epilepsy Res 110: 62-70, 2015.

27. Harden C, Tomson T, Gloss D, Buchhalter J, Cross JH, Donner E, French JA, Gil-Nagel A, Hesdorffer DC, Smithson WH, et al: Practice guideline summary: Sudden unexpected death in epilepsy incidence rates and risk factors: Report of the guideline development, dissemination, and implementation subcommittee of the American academy of neurology and the American epilepsy society. Neurology 88: 1674-1680, 2017.

28. Mehvari Habibabadi J, Badihian S, Tabrizi N, Manouchehri N, Zare $M$, Basiratnia $R$, Barekatain $M$, Moein $H$, Mehvari Habibabadi A, Moein P and Gookizadeh P: Evaluation of dual pathology among drug-resistant epileptic patients with hippocampal sclerosis. Neurol Sci 40: 495-502, 2019.

29. Wang S, Jin B, Aung T, Katagiri M, Jones SE, Krishnan B, Gonzalez-Martinez JA, Prayson RA, Najm IM, Alexopoulos AV, et al: Application of MRI post-processing in presurgical evaluation of non-lesional cingulate epilepsy. Front Neurol 9: 1013, 2018.

30. Colon AJ, Osch MJPV, Buijs M, Grond JVD, Hillebrand A, Schijns O, Wagner GJ, Ossenblok P, Hofman P, Buchem MAV and Boon P: MEG-guided analysis of 7T-MRI in patients with epilepsy. Seizure 60: 29-38, 2018.

31. Jin B, Krishnan B, Adler S, Wagstyl K, Hu W, Jones S, Najm I, Alexopoulos A, Zhang K, Zhang J, et al: Automated detection of focal cortical dysplasia type II with surface-based magnetic resonance imaging postprocessing and machine learning. Epilepsia 59: 982-992, 2018.

32. Alshafai L, Ochi A, Go C, McCoy B, Hawkins C, Otsubo H, Snead OC, Rutka J and Widjaja E: Clinical, EEG, MRI, MEG, and surgical outcomes of pediatric epilepsy with astrocytic inclusions versus focal cortical dysplasia. Epilepsia 55: 1568-1575, 2014.

33. Najafi MR, Malekian M, Akbari M and Najafi MA: Magnetic resonance imaging and electroencephalography findings in a sample of Iranian patients with epilepsy. J Res Med Sci 23: 106, 2018.

34. Torres CV, Pastor J, Garcia-Navarrete E, Pulido-Rivas P and Sola RG: Classification of structural lesions in magnetic resonance imaging. Surgical implications in drug-resistant epilepsy patients. Rev Neurol 61: 241-248, 2015 (In English, Spanish).

35. Peng SJ, Harnod T, Tsai JZ, Ker MD, Chiou JC, Chiueh H, Wu CY and Hsin YL: Evaluation of subcortical grey matter abnormalities in patients with MRI-negative cortical epilepsy determined through structural and tensor magnetic resonance imaging. BMC Neurol 14: 104, 2014.

36. van Rooijen BD, Backes WH, Schijns OE, Colon A and Hofman PA: Brain imaging in chronic epilepsy patients after depth electrode (stereoelectroencephalography) implantation: Magnetic resonance imaging or computed tomography? Neurosurgery 73: 543-549, 2013.

37. Pizarro R, Nair V, Meier T, Holdsworth R, Tunnell E, Rutecki P, Sillay K, Meyerand ME and Prabhakaran V: Delineating potential epileptogenic areas utilizing resting functional magnetic resonance imaging (fMRI) in epilepsy patients. Neurocase 22: 362-368, 2016

This work is licensed under a Creative Commons Attribution-NonCommercial-NoDerivatives 4.0 International (CC BY-NC-ND 4.0) License. 